\title{
An Analysis of Ground-Based Polarimetric Sky Radiance Measurements
}

\author{
B. Cairns \\ Columbia University \\ New York, New York \\ B. E. Carlson and A. A. Lacis \\ NASA/Goddard Institute for Space Studies \\ New York, New York \\ E. Russell \\ Spectir Corporation \\ Goleta, California
}

\section{Abstract}

An analysis of polarimetric sky radiance measurements made with the engineering model of the Galileo photopolarimeter/radiometer at wavelengths of $410 \mathrm{~nm}$ and $678 \mathrm{~nm}$ is presented. The polarization measurements are accurate to within $0.1 \%$, while the radiance measurements have no absolute calibration, but are linear over a wide dynamic range. The measurements analyzed are almucantar scans made in Goleta, California, which has a well-defined marine boundary layer. A two-layer model of the atmosphere was therefore used in which a Rayleigh scattering gas layer is assumed to overlie an aerosol layer. An initial estimate of the aerosol model is obtained by finding the best fit of the polarization and intensity data at both wavelengths to a precalculated data base. Preliminary results indicate that if the fit is inadequate, an iterative inversion can be used in which a size distribution with some size resolution of refractive index is obtained.

\section{Experimental Measurements}

An "engineering spare" of the Galileo Photopolarimeter /Radiometer (PPR) measures the state of polarization to within $0.1 \%$ for wavelengths of $410 \mathrm{~nm}, 678 \mathrm{~nm}$ and $945 \mathrm{~nm}$. Although lacking an absolute calibration, the sky radiance measurements nonetheless provide useful information about the microphysical properties of aerosols since the response of the instrument is linear to better than $1 \%$ over its entire dynamic range. This instrument's instantaneous field of view, provided by a Cassegrainian Dall-Kirkham telescope, is 0.14 degrees. A Wollaston prism is used to produce two spatially separated orthogonal polarization states which are measured simultaneously. Half wave retarders are positioned to produce $\mathrm{x}+0_{-}, \mathrm{x}+45_{-}$and $\mathrm{x}+90_{-}$in successive measurements. The first two retarder positions allow the degree and plane of polarization to be evaluated, while the third measurement provides a check on scene stability and detector gain by interchanging the roles of the two detectors as compared to the first retarder position.

The measurements taken using this instrument are almucantar scans that are truncated before the direct solar beam is reached. The measurements are acquired using a PC, and each measurement cycle of 23 seconds is time-stamped so that the movement of the sun during the course of data acquisition can be included in the model calculation. The data were acquired for solar and instrument elevation angles between 25 and 30 degrees over a number of days in April, May, and October of 1996 in Santa Barbara, California. Data were taken on days that were very clear and also on a number of days when the sky was contaminated by smoke from fires some 60 miles away.

\section{Modeling}

The use of polarimetric measurements for remote sensing has been appreciated for a long time (Herman et al. 1971, Hansen and Hovenier 1974). One problem with the interpretation of polarimetric data has been the difficulty in calculating the Stokes vector for a realistic atmosphere for a diverse set of microphysical and atmospheric properties, mostly because such calculations are time-consuming. Recent advances in computational speed and algorithmic improvements (de Haan et al. 1987) now allow the rapid computation of the scattering properties of a large set of aerosol and surface models, with a prescribed accuracy. 
The downwelling radiation was calculated using a vector doubling/adding calculation (Hansen and Travis 1974) with a number of modifications related to improved computational efficiency. The Fourier expansion coefficient of the azimuthal variation beyond which the full doubling calculation can be terminated and replaced by first and second order scattering calculations can be evaluated automatically, with almost no computational penalty, by comparing the second order calculation with the full doubling result for each term in the azimuthal decomposition. The full doubling calculation can then be replaced with a second order calculation while maintaining a specified level of accuracy. The fact that error growth is linear with optical depth for the type of small optical depths relevant to aerosol retrievals can be used to estimate the required initial thickness of the layer to start the doubling calculation. This is done by performing the calculation for the first azimuth term with an extremely thin (conservative) value of the starting optical thickness.

The doubling calculation is initiated with a second order scattering calculation. As the layer is doubled from the initial thickness, the error that would have been made if the calculation had been started at that thickness is calculated. The required starting thickness can be evaluated by estimating the growth of this error to ensure that a prescribed final error is achieved. This starting thickness can then be used in subsequent doubling calculations with confidence that the required starting thickness for the first term in the azimuthal decomposition is a conservative estimate of that required for higher order terms.

Finally the single scattering contribution was treated separately (cf. de Haan et al. 1987). This allows the single scatter contribution to be evaluated at the exact points at which data are acquired, minimizing errors that would be introduced through interpolation in zenith angle or the computational burden that would be incurred by increasing the number of zenith gauss points.

The atmospheric model we used was one in which a Rayleigh scattering gas layer overlies an aerosol layer with a Lambertian surface underneath the atmosphere. This is a particularly good assumption for the measurements that we analyze here because Goleta has a well-defined marine boundary layer. It should also be noted that, provided almucantars are acquired with an instrument elevation of greater than 20 degrees, the error introduced by erroneous assumption about vertical structure is small. Separating the molecular scattering and the aerosol scattering into two layers also has the advantage that a range of aerosol optical thicknesses can be rapidly calculated by adding to an initial thin aerosol layer. The assumption that the surface was a Lambertian reflector may not be an adequate approximation for downward-looking instruments; but for upward-looking instruments, we found that the dominant property of the surface that affected the downwelling radiation was the albedo. The inclusion of specular reflection from randomly oriented facets (for example, leaves or soils) for a fixed albedo had only a weak effect on either the sky radiance distribution or the polarization of the downwelling radiation.

The use of the computational methods noted above applied to the simple two-layer scattering atmosphere above a Lambertian surface assumed here allows a full vector doubling/adding calculation in 4 seconds on a standard engineering workstation. This speed allows us to calculated the sky radiance distribution for a wide range of aerosol and surface properties.

The calculations are performed for the 410-nm and 678-nm bands of the PPR because accurate modeling of the 945-nm band, which overlaps a strong water vapor absorption feature, would require correlative information about the water vapor profile. The aerosol scattering properties were obtained from Mie scattering calculations which used a gamma size distribution, with a fixed effective variance of 0.1 (Hansen and Travis 1974), while the refractive index was allowed to vary between 1.35 and 1.55 in steps of 0.05 . The effective radius was varied between 0.1 and $0.6 \mu \mathrm{m}$ in $0.1 \mu \mathrm{m}$ steps. The optical depth referenced to a 550-nm wavelength was varied between 0.02 and 0.1 in steps of 0.02 , the size of the aerosol defining the optical depth at the wavelengths of interest.

The degree and plane of polarization as well as the sky radiance data, at the two wavelengths, are used in estimating which model is best. Since the sky radiance measurements are uncalibrated, they are scaled to provide a best fit to each model and then the deviation of the model from the scaled measurement is used in evaluating which model has the best RMS fit to the data.

On 12 April 1996, which was a clear day, the best fit to the data we obtained was for an effective radius of $0.3 \mu \mathrm{m}$, a refractive index of 1.5, an optical depth (@550 nm) of 0.06, and a surface albedo of 0.1. The inferred surface albedo is consistent with both 1-km-resolution advanced very high resolution radiometer (AVHRR) Channel 1 reflection measurements for April 1992 and the spring season climatological average 6-km-resolution data for 1985-1988. It should be noted that when the sky radiance data only are used in the fit, a model that has an effective radius of $0.3 \mu \mathrm{m}$, a refractive index of 1.45, an optical depth (@550 nm) of 0.04 , and a surface albedo of 0.05 provides the best fit.

On 21 October 1996 (a clear day for which $410 \mathrm{~nm}$ maximum polarization was greater than $70 \%$ ), the best fit to the data was 
for an effective radius of $0.5 \mu \mathrm{m}$, a refractive index of 1.4 , an optical depth (@550 nm) of 0.02, and a surface albedo of 0.15. This inferred surface albedo is consistent with AVHRR measurements for October. When only the sky radiance data are used in the fit, a model that has an effective radius of $0.5 \mu \mathrm{m}$, a refractive index of 1.5, an optical depth (@550 nm) of 0.02 , and a surface albedo of 0.05 provides the best fit.

We also obtained data for a period, 1 May 1996, when forest fires were burning in California, producing large, relatively uniform clouds of smoke that were visible to the naked eye. The best fit to this data has an effective radius of $0.2 \mu \mathrm{m}$, refractive index of 1.5 , optical depth (@550 nm) of 0.08 , and a surface albedo of 0.15 . The best fit obtained using only the sky radiance data is a model that has an effective radius of $0.2 \mu \mathrm{m}$, a refractive index of 1.45, an optical depth (@550 nm) of 0.06 , and a surface albedo of 0.05 . The fact that in the best fit using all the data, the inferred surface albedo is higher than that obtained for 12 April may indicate that a background aerosol is present and that a multimodal aerosol model is more appropriate for these data. The inferred size is consistent with other measurements of smoke from forest fires (Radke et al. 1978), and the refractive index is consistent with that estimated using polar nephelometer measurements of smoke particles (Veretennikov et al. 1980).

Figures 1, 2, and 3 show the fidelity of the fit of the model to the data when all the data are used (Figure 1); when only polarization measurements are used (Figure 2); and when only intensity measurements are used (Figure 3). The particular case shown is for the smoky sky case of 1 May 1996, which was the worst fit we obtained with our brute force method.

\section{Conclusions}

Evidently the aerosol model used here is very simple, but given that the data were taken without corroborative sunphotometry or lidar measurements, one could easily overfit the data and draw unwarranted conclusions. Nonetheless, the results obtained thus far are robust. For example, it is not possible for the dominant size mode of the smoke particles to be any larger than 0.2 microns because the maximum polarization for the 678-nm channel was greater than that for the 410-nm channel which can only occur for small particles. Preliminary results suggest that a method similar to that applied by Nakajima et al. (1983) to radiance data can be applied to polarimetric data to obtain a size- and refractiveindex-resolved distribution of aerosol number densities.

It is also clear from analyzing single scattering inversions that the uncertainties in the retrievals of large particles from radiance data are substantially reduced by the inclusion of polarimetric measurements. It is therefore apparent that polarimetry is a useful tool in evaluating aerosol properties, particularly the refractive index which would allow the identification of aerosol type.

The use of polarimetric data would be even more powerful when used in conjunction with other measurements, such as those from a shadowband radiometer (Harrison et al. 1994) that measures both the direct and the diffuse components of downwelling solar radiation. This would provide strong constraints on the single scatter albedo, the surface albedo, and the size distribution (King and Herman 1979). Although the PPR is only of use for intensive observation periods, because it requires considerable human intervention in the acquisition of data, polarization measurements are currently been acquired by CIMEL, a multispectral sun and sky radiometer, (Holben et al. 1997) in many locations around the world. The understanding obtained in reducing and analyzing the PPR data should therefore be of great use in making the best use of this large and, hopefully, long-term data set. 


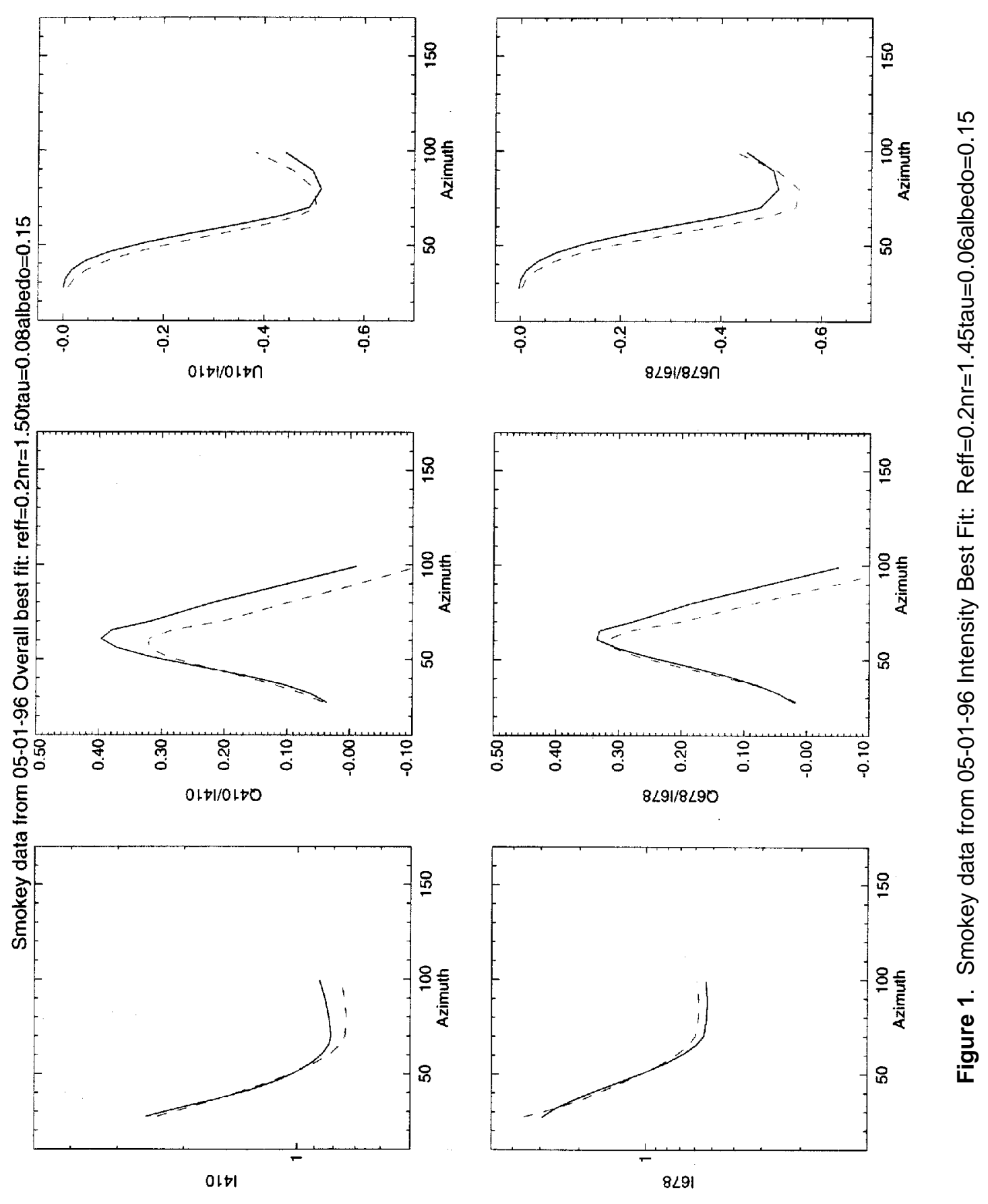









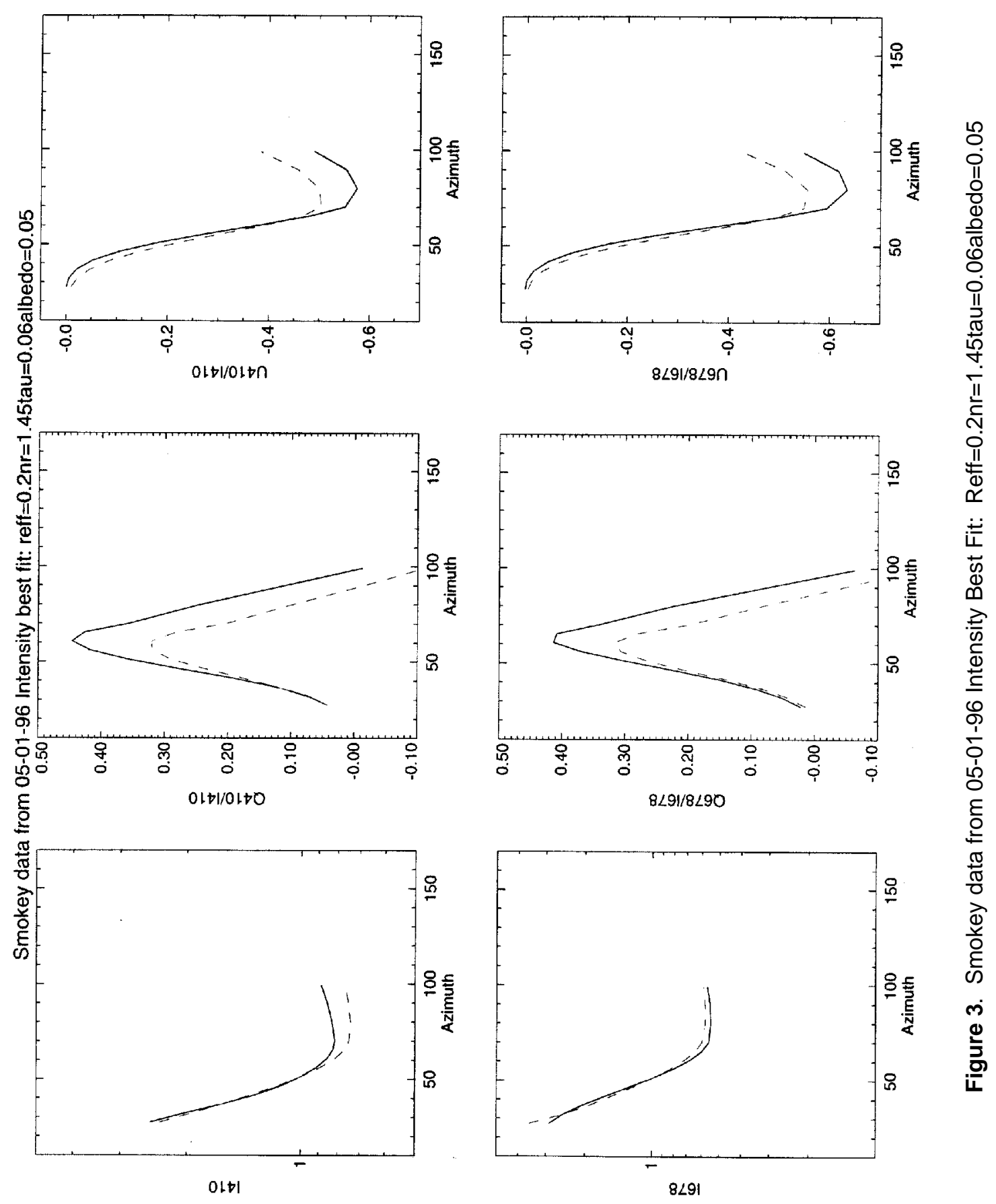




\section{References}

de Haan, J. F., P. B. Bosma and J. W. Hovenier, 1987: The adding method for multiple scattering calculations of polarized light. Astronom. Astrophys., 183, 371.

Hansen J. E., and J. W. Hovenier, 1974: Interpretation of the polarization of Venus. J. Atmos. Sci., 31, 1137-1160.

Hansen, J. E., and L. D. Travis, 1974: Light scattering in planetary atmospheres. Space Sci. Rev., 16, 527-610.

Harrison, L., J. Michalsky, and J. Berndt, 1994: Automated multifilter rotation shadow-band radiometer: An instrument for optical depth and radiation measurements. Appl. Opt., 33, 5118-5125.

Herman, B. M., S. R. Browning, and R. J. Curran, 1971: The effects of atmospheric aerosols on scattered sunlight. J. Atmos. Sci., 28, 419-428.

Holben, B. N. et al., 1997: Automatic sun and sky scanning radiometer system for network aerosol monitoring, submitted to Rem. Sens. Env., (also available from http://spamer.gsfc.nasa.gov).
King, M. D., and B. E. Herman, 1979: Determination of the ground albedo and the index of absorption of atmospheric particulates by remote sensing. Part I: Theory. J. Atmos. Sci., 36, 163-173.

Nakajima, T., M. Tanaka, and T. Yamauchi, 1983: Retrieval of the optical properties of aerosols from aureole and extinction data. Appl. Opt., 22, 2951-2959.

Radke, L. F., J. L. Stith, D. A. Hegg, and P. V. Hobbs, 1978: Airborne studies of particles and gases from forest fires. $J$. Air Poll. Control Assoc., 28, 30-34.

Veretennikov, V. V, V. S. Kozlov, I. E. Naats, and V. Ya Fadeyev, 1980: On the determination of the optical constants and microstructure of smoke aerosols from optical polarization measurements. Izv. Atmos. Ocean. Phys., 16, 177-181 